\title{
La Coparticipación de los Jueces Comunitarios en la Ad- ministración de Justicia
}

\section{Joint Participation of Community Judges in the Adminis- tration of Justice}

Carlos A. de Tomaso R. ${ }^{a}$

\author{
INFORMACIÓN DEL \\ ARTÍCULO \\ Fecha de recepción: 11 de enero del \\ 2017 \\ Fecha de aceptación: 15 de junio de \\ 2017
}

\begin{abstract}
Resumen
El trabajo tiene como objetivo analizar la aplicación de las normas comunitarias andinas y la coparticipación de jueces comunitarios en el ejercicio de la competencia de la administración de justicia; en ese contexto se analizará la competencia de interpretación prejudicial del Tribunal Andino de Justicia, de tal forma de clarificar su ámbito, finalidad y su ejercicio y con una demostración jurisprudencial relativa a marcas.
\end{abstract}

\section{Palabras Clave:}

Jueces comunitarios, justicia, competencia, ámbito, marcas. Clasificación JEL: K100

\begin{abstract}
The purpose of this work is to analyze the application of Andean community rules and the participation of community judges in the exercise of the competence of the administration of justice; The jurisdiction of the Andean Court of Justice will be analyzed in this context so as to clarify its scope, its purpose and its exercise and with a legal demonstration regarding trademarks.
\end{abstract}

\section{Keywords}

Community judges, justice, competence, scope, trademarks. JEL Classification: K100 


\section{Introducción}

Llegará un día en que tú, Francia; tú, Rusia; tú Inglaterra; tu, Alemania; todas vosotras, naciones del continente, sin perder vuestras cualidades distintas y vuestra gloriosa individualidad, os fundiréis estrechamente en una unidad superior y constituiréis la fraternidad europea, igual que se han fundido Normandía, Bretaña, Borgoña, Lorena, Alsacia, todas nuestras provincias en Francia. Llegará un día en que no habrá más campos de batalla que los mercados abriéndose al comercio y los espíritus abriéndose a las ideas... Llegará un día en veremos a esos grupos inmensos, los Estados Unidos de América y los Estados Unidos de Europa, colocados frente a otro, tendiéndose la mano por encima de los muertos, intercambiando productos, su comercio, su industria, su arte, sus genios (...) (Víctor Hugo -siglo XIX- París 21 de agosto 1849 en Diez Moreno, 2009, p.30 ).

El pensamiento de Víctor Hugo nos demuestra que la idea de integración no es de hoy, fue de siempre, con las salvedades de los pensamientos de cada época. Los denominados procesos de integración son protagonistas en el mundo actual, es una corriente imparable! Pocos son los actores sociales que niegan ese camino como el trayecto hacia el desarro1lo económico y social, talvez teniendo como único argumento válido los acostumbrados abusos de las grandes potencias mundiales, sin perjuicio de lo cual -a mi criterio- justamente los procesos de integración tienen como uno de sus obje- tivos el acortar distancias uniendo las potencialidades de los países en desarrollo de tal forma de equiparar las condiciones de negociación internacional.

Dentro de ese escenario, es indudable que los procesos de integración implican afectaciones a los sistemas jurídicos nacionales, toda vez que al expedirse -mediante tratados, decisiones u otros instrumentos- legislación comunitaria, el ámbito del derecho crece y se vuelve más complejo, presentándose no sólo problemas de jerarquía e incorporación normativa sino además la participación de nuevos actores en la aplicación de las normas y la administración de justicia.

Justamente, el presente trabajo tiene como objetivo analizar la aplicación de las normas comunitarias andinas y la coparticipación de Jueces comunitarios en el ejercicio de la competencia de la administración de justicia.

El capítulo primero del presente trabajo, tendrá como objetivo el estudio de la competencia de Interpretación Prejudicial del Tribunal Andino de Justicia, de tal forma de clarificar su ámbito, finalidad y su ejercicio.

En el capítulo segundo, analizaré un fallo de Interpretación Prejudicial del Tribunal de Justicia de la CA, de una marca notoriamente conocida, de tal forma de explorar el ejercicio práctico de esta cooperación jurisdiccional y el alcance de la misma en un caso concreto.

Finalizaré, con las conclusiones derivadas del Estudio del presente trabajo, 
que aspiro que contribuyan al foro jurídico en la discusión de la aplicabilidad del derecho comunitario.

El trabajo se ha basado en el estudio de doctrina sudamericana y europea, normas comunitarias andinas y europeas, y obviamente de varios fallos del Tribunal de Justicia de la Comunidad Andina, que me sirvieron para clarificar la aplicación de los conceptos y poder hacer el análisis adecuado del tema.

\section{La Interpretación Prejudicial del Tribunal de Justicia del Acuerdo de Cartagena}

\section{Antecedentes.}

El profesor Rengifo García sostiene que:

El derecho comunitario andino se caracteriza por ser autónomo, tener efecto coercitivo; integrarse a los ordenamientos jurídicos nacionales, en donde sus efectos son directos; prevalecer sobre el derecho de los Estados Miembros; representar un derecho único para toda la comunidad, y ser de aplicación uniforme (1996).

Este adecuado criterio nos sirve para introducirnos al análisis del recurso de Interpretación prejudicial en la Comunidad Andina, toda vez que del estudio se desprenderá la confirmación de los elementos de este criterio.
Casi de manera unánime se sostiene que la interpretación prejudicial tiene su origen en Europa, con el Tratado de la Comunidad Europea (conocido como Tratado de Roma) ${ }^{1}$, conforme lo sustentan los profesores Mangas Martín y Liñan Nogueras quienes resaltan que la función encomendada al Tribunal de Justicia de la Unión Europea es la de "garantizar el respeto del Derecho en la interpretación y aplicación del tratado de la Comunidad" $\mathrm{y}$ por tanto, los jueces comunitarios deben compartir el ejercicio de la función jurisdiccional con los órganos jurisdiccionales de los países miembros que son los llamados a aplicar en primera instancia las normas comunitarias, debiéndose convertir en los jueces ordinarios del Derecho Comunitario.

El Profesor Alejandro Daniel Perotti sostiene que los procesos de integración requieren a fin de asegurar el imperio del Derecho en el bloque, y con ello una garantía para la seguridad jurídica y la efectiva salvaguarda de los derechos de los particulares, el establecimiento de un sistema jurisdiccional, que no sólo controle la legalidad de las normas de las instituciones comunitarias y de aquellas que sean producto de la potestad legislativa de los Estado, sino también la compatibilidad de las conductas de las personas físicas y jurídicas (que desarrollan su actividad dentro del espacio regional) con el ordenamiento comunitario.

En este sentido, al crearse un sistema

${ }^{1}$ Suscrito el 25 de marzo de 1957 (Comisión Europea, 2000). 
jurídico comunitario, se da por entendido que habrá órganos supranacionales con competencias para establecer normas jurídicas y procedimientos de cumplimiento obligatorio para los países miembros, y conforme lo sostiene el profesor Diez Moreno (2009) la normativa comunitaria tiene consecuencias inmediatas por cuanto se entiende plenamente integrada al ordenamiento interno de los Estados, las mismas que deben ser aplicadas de manera directa -salvo que se disponga lo contrario- y tienen primacía sobre las normas ordinarias -salvo las constitucionales- por lo que cualquier norma contradictoria debe ser inaplicada. Estas características de las normas comunitarias han sido reconocidas por el Tribunal de Justicia de la Comunidad Andina (en adelante TJCA) ${ }^{2}$ al declarar "(...) debe tenerse en cuenta el principio de la primacía de las normas comunitarias y el de la aplicación directa de las mimas (...)"

Así, siguiendo el esquema europeo, la Comunidad Andina ${ }^{3}$ estableció la herramienta de cooperación entre el juez nacional y la jurisdicción supranacional, a través del establecimiento de la figura de la interpretación prejudicial. El Tribunal de Justicia de la Comunidad Andina $^{4}$, declara que en virtud de la aplicabilidad inmediata y directa de las normas comunitarias en el territorio de los Países Miembros, los particulares pueden invocarlas ante sus jueces nacionales. De esta característica, surge la ne- cesidad de contar con un mecanismo que garantice que la aplicación e interpretación del Derecho andino por parte de los distintos jueces nacionales se realicen de manera uniforme en los cinco Países Miembros.

\section{Definición.}

El profesor Guy Isaac (1995) sostiene que la Interpretación Prejudicial es un mecanismo de cooperación judicial por el cual el órgano jurisdiccional nacional y el Tribunal de Justicia, en el orden de sus propias competencias, son llamados a contribuir directa y recíprocamente en la elaboración de una decisión”.

El artículo 32 del Tratado de Creación del TJAC (modificado por el protocolo de suscrito en Cochabamba, Bolivia) establece: "Corresponderá al Tribunal interpretar por vía prejudicial las normas que conforman el ordenamiento jurídico de la Comunidad Andina, con el fin de asegurar su aplicación uniforme en el territorio de los países miembros".

Conforme lo sostiene el profesor doctor Klaus-Dieter Borchandt, en el sistema europeo tiene la interpretación prejudicial tiene el mismo objetivo de garantizar una aplicación uniforme del derecho comunitario y destacan la importancia de esta figura para la protección de los derechos individuales.

\footnotetext{
${ }^{2}$ Proceso 5 IP-89, sustanciado ante el TJAC

${ }^{3}$ El Acuerdo de Cartagena, fue suscrito el 25 de mayo de 1969. Posteriormente se han suscrito varios Protocolos Modificatorios

${ }^{4}$ El artículo 6 del Acuerdo de Cartagena establece que el TJAC es un órgano del Sistema Andino de Integración.
} 
Ratificando lo antes expuesto, dentro del proceso 35-IP-2000, el TJCA sostuvo:

Que el Tribunal de lo Contencioso Administrativo, Distrito de Quito, es competente para solicitar la interpretación prejudicial de normas que conforman el Ordenamiento Jurídico del Acuerdo de Cartagena, por cuanto ante ese alto organismo jurisdiccional ecuatoriano se ventila un proceso en el que deben ser aplicadas tales normas comunitarias. Que este Tribunal es competente para interpretar en vía prejudicial las normas que conforman el Ordenamiento Jurídico siempre que la solicitud provenga de un Juez Nacional actuando en función de Juez Comunitario, como lo es en este caso la alta jurisdicción consultante, $\mathrm{y}$ en tanto y en cuanto aquellas resulten pertinentes, a juicio del Tribunal Andino para la resolución del litigio interno (p. 89).

\section{Las normas del Ordenamiento Jurí- dico Andino.}

Vistos, que el Tratado de Creación del TJAC le otorga la competencia para interpretar por vía prejudicial las normas que conforman el ordenamiento jurídico de la Comunidad Andina, resulta evidente la necesidad de identificarlas.

Con acierto, en el artículo primero del Tratado de Creación del TJAC ${ }^{5}$ se enu- meran taxativamente las normas que conforman el antes citado ordenamiento, a saber:

a) El Acuerdo de Cartagena, sus Protocolos e Instrumentos adicionales;

b) El presente Tratado y sus Protocolos Modificatorios;

c) Las Decisiones del Consejo Andino de Ministros de Relaciones Exteriores y la Comisión de la Comunidad Andina;

d) Las Resoluciones de la Secretaría General de la Comunidad Andina; y,

e) Los Convenios de Complementación Industrial y otros que adopten los Países Miembros entre sí y en el marco del proceso de la integración subregional andina.

En tal sentido, a través de esta norma se puede tener con claridad el ámbito de la competencia asignada al TJCA.

\section{La Finalidad de la Interpretación Prejudicial.}

Tal como se desprende del artículo 34 del Tratado de Creación del TJCA, la finalidad de la interpretación es precisar el contenido y alcance de las normas que conforman el ordenamiento jurídico de la comunidad andina garantizando la aplicación uniforme de dichas normas. Le está prohibido al Tribunal interpretar el alcance y contenido de las normas nacionales de los Estados miembros, así como calificar los hechos materia del proceso, sin

\footnotetext{
${ }^{5}$ Modificado por el Protocolo de Cochabamba.
} 
perjuicio de que pueda referirse a estos cuando sea indispensable ad luz de la interpretación.

En ese sentido, queda clara la finalidad de la interpretación como quedó claro sobre qué normas tiene ámbito este recurso.

Sobre este punto, quiero hacer un paréntesis para mencionar que en el sistema europeo, la finalidad de la interpretación prejudicial es más amplia, toda vez que no sólo tiene competencia para la interpretación de las normas comunitarias sino también para interpretar los actos adoptados por las Instituciones de la Comunidad Europea y pronunciarse sobre su validez. Por eso, el profesor Diez Moreno (2009, p. 297) sostiene que existen dos clases de cuestiones prejudiciales en la comunidad europea: la interpretativa y la de validez, en la una se interpreta una norma comunitaria cuya validez no se discute; en la otra, afecta a la legalidad o ilegalidad del Derecho comunitario europeo, ya que si la cuestión se resuelve negativamente, se cierra la posibilidad de aplicación posterior del acto sujeto a análisis. Sostiene además el profesor Diez, que a su criterio la interpretación no es propiamente un recurso, sino un procedimiento de cooperación entre el Juez comunitario y los tribunales nacionales, criterio que en cierta forma ha sido acogido por el TJAC ${ }^{6}$, quien ha declarado:

(...) entre las características del Trata- do de Creación del Tribunal, se destaca la de haber sido privada la interpretación prejudicial de la naturaleza y del calificativo de acción, que el Tratado le ha otorgado en cambio a las otras dos vías de acceso al Tribunal Andino: las acciones de nulidad y de incumplimiento (...).

Esa distinción del sistema europeo, también es citada por el doctor Klaus-Dieter Borchandt (1999), quien sostiene que el objeto de las cuestiones prejudiciales en la comunidad europea son por una parte la interpretación del derecho y por otra el "control de validez" sobre los actos de las instituciones comunitarias.

El Estatuto ${ }^{7}$ del TJCA, a su vez establece en el artículo 121:

Corresponde al Tribunal interpretar las normas que conforman el ordenamiento jurídico de la Comunidad Andina, con el fin de asegurar su aplicación uniforme en el territorio de los Países Miembros.

Por tanto, no podemos dejar de lado que la interpretación prejudicial persigue y se justifica en el sentido de asegurar la aplicación unirme de las normas comunitarias andinas.

Respecto de los métodos interpretativos que debe aplicar el TJAC, si bien ni en el Tratado ni en el Estatuto se hace re-

${ }^{6}$ Proceso 11-IP-96, TJAC

${ }^{7}$ Decisión 500, del Consejo de Ministros del Acuerdo de Cartagena 
ferencia, a mi criterio los magistrados deben aplicar los reconocidos por la Filosofía en general y en especial por la Filosofía y Hermenéutica Jurídica, métodos tales como el gramatical, exegético, histórico, sistemático, lógico jurídico, etc, ampliamente desarrollados por la doctrina, toda vez que sólo a través de todos ellos se podrá encontrar el verdadero sentido de las normas dentro de un sistema jurídico.

\section{Efectos de la Interpretación Preju- dicial.}

En la doctrina europea estudiada, se discute la necesidad o no de que las interpretaciones prejudiciales de normas comunitarias tengan efecto "erga omnes". Quienes se inclinan positivamente, sostienen que del estudio de los casos las normas interpretadas concluyen que son las mismas normas las interpretadas en gran parte de los casos, por lo que se produce una congestión innecesaria en los Tribunales comunitarios. A su vez, los que se inclinan negativamente, sostiene que al ser prejudicial, es decir que nacen de un proceso en particular, la interpretación de la norma sólo puede ser aplicada al caso concreto, toda vez que cada caso tiene sus propias particularidades. El artículo 35 del Protocolo Modificatorio de Cochabamba del TJAC, estable que el Juez que conozca el proceso deberá de manera obligatoria adoptar en su sentencia la interpretación del Tribunal.

Es evidente que la Comunidad Andina no adoptó la tesis de que la interpretación tenga efectos obligatorios generales, ya que el único obligado a su acatamiento es el Juez nacional que conoce el proceso donde se ha suscitado el conflicto que requiere de interpretación normativa. Obviamente, las interpretaciones prejudiciales sirven como precedentes jurídicos, aunque no son obligatorios, salvo que formen parte de la motivación de una nueva interpretación prejudicial.

En el sistema europeo, conforme lo sostiene el doctor Klaus-Dieter Borchandt (1999, p.91) la decisión prejudicial obliga al tribunal solicitante y a todos los demás órganos que se ocupan del litigio correspondiente, y reviste de un efecto prejudicial considerable para otros procedimientos similares.

A mi criterio, podría establecerse un sistema mixto: por un lado mantener el sistema de interpretación prejudicial actual pero paralelamente establecer la competencia del TJAC de interpretar de oficio, y con carácter generalmente obligatorio, cualquier norma comunitaria cuyo sentido no sea claro y cause controversia.

En este punto, también considero necesario mencionar los países miembros de la Comunidad Andina se obligaron a velar por el cumplimiento de las disposiciones del Tratado de Creación del TJCA y en particular al cumplimiento de los jueces nacionales a las interpretaciones prejudiciales emitidas por el $\mathrm{TJCA}^{8}$. Conforme al Estatuto del TJCA, la no a-

${ }^{8}$ Obligación establecida en el artículo 36 del Tratado de Creación del TJCA 
plicación de la interpretación puede ser el fundamento para iniciar un recurso de incumplimiento.

\section{Clases de Interpretación Prejudi- cial en la Comunidad Andina.}

Del análisis del artículo 33 del Tratado de Creación del TJCA, se desprende que existen dos clases de interpretación prejudicial: a) La obligatoria; y, b) la Facultativa.

Sobre el tema, el profesor Perotti (2001), sostiene:

En la Comunidad Andina la interpretación prejudicial se distingue, según el juez del cual deba proceder la consulta, en "obligatoria" y "facultativa" (art. 33 TCTJ). La primera comprende todos aquellos supuestos en los que la decisión del tribunal nacional, que aplica derecho comunitario andino, no sea susceptible de ser atacada por recurso judicial, según las disposiciones del derecho interno. En estas circunstancias el juez se encuentra constreñido a realizar el reenvío al Tribunal de Justicia. En cambio, es facultativa siempre que contra dicha sentencia del juez nacional existan -según el ordenamiento interno- vías recursivas por las cuales sea posible su cuestionamiento. Frente a dicha hipótesis es discrecional la decisión del juez de consultar al Tribunal de Quito (TJCA). Es decir, que el factor diferenciador está dado por si en el proceso sustanciado ante el juez o el Tribunal nacional, existe la posibilidad de presentar algún recurso, o en otras palabras, que no sea la instancia definitiva (p. 2).
Sobre la consulta Facultativa, el artículo 122 del Estatuto del TJCA, establece que los jueces nacionales que conozcan de un proceso en el que deba aplicarse o se controvierta alguna de las normas que conforman el ordenamiento jurídico de la Comunidad Andina, podrán solicitar, directamente y mediante simple oficio, la interpretación del Tribunal acerca de dichas normas, siempre que la sentencia sea susceptible de recursos en derecho interno. Si llegare la oportunidad de dictar sentencia sin que hubiere recibido la interpretación del Tribunal, el juez deberá decidir el proceso. A su vez, sobre la consulta obligatoria el mismo Estatuto establece en su artículo 123 que de oficio o a petición de parte, el juez nacional que conozca de un proceso en el cual la sentencia fuera de única o última instancia, que no fuere susceptible de recursos en derecho interno, en el que deba aplicarse o se controvierta alguna de las normas que conforman el ordenamiento jurídico de la Comunidad Andina, deberá suspender el procedimiento y solicitar directamente y mediante simple oficio, la interpretación del Tribunal.

Sería fundamental para efectos de la aplicación clara de estas normas que el órgano correspondiente de la Comunidad Andina aclare que se entiende por "recurso" al amparo de la normatividad andina, caso contrario podría provocar oscuridad al momento de decidir si es o no obligatoria la interpretación en un caso específico. Al respecto el profesor doctor Klaus-Dieter Borchandt (1999, p. 91) sostiene que para cuestiones prejudiciales en Europa, el término "Recurso" comprende todos los remedios jurídicos que 
permiten que una decisión adoptada por un tribunal pueda ser revisada por una instancia judicial superior por motivos de hecho y derecho. No comprende, agrega, los remedios jurídicos ordinarios con efectos limitados y específicos (como el recurso de revisión o acción de insconstitucionalidad). Aquí entonces una luz, a la duda que he planteado.

También mencionar que de conformidad con el artículo 33 del Tratado de Creación y del 124 del Estatuto del TJCA, en el caso de la consulta obligatoria, el proceso se deberá suspender en su tramitación por parte del juez nacional hasta tanto reciba la interpretación prejudicial.

\section{La Legitimación Activa y Pasiva del Recurso de Interpretación Prejudi- cial.}

Del artículo 33 del Tratado de Creación del TJCA también se extrae que la legitimación activa de la interpretación prejudicial la tiene de manera exclusiva el Juez nacional que haya prevenido en el conocimiento de la causa en ejercicio de sus atribuciones jurisdiccionales, por lo que quedan descartados jueces no competentes (nacionales o extranjeros) así como las autoridades y órganos de gobierno (incluidas las legislativas) de los Estados miembros.

Sin embargo debo indicar que el TJCA, en ejercicio de sus competencias negó la solicitud de interpretación prejudicial solicitada por el Instituto de Defensa y Competencia y del Protección de la Propiedad Industrial en Perú, por no pertenecer a la estructura jurisdiccional ${ }^{9}$ pero si ha aceptado en varias oportunidades solicitudes del Consejo de Estado de Colombia (órgano ubicado por varios tratadistas dentro del ámbito administrativo). En este sentido, se puede apreciar que el TJCA realiza interpretaciones relativas a qué órganos conforman la estructura jurisdiccional de los Estados miembros.

Sobre este punto, el doctor Klaus-Dieter Borchandt (1999), sostiene que a nivel de la comunidad europea, a efectos de la interpretación prejudicial, debe entenderse por órgano jurisdiccional todas las instituciones independientes, es decir, no sujetas a instrucciones, que deben adoptar resoluciones en un procedimiento regulado por el ordenamiento jurídico nacional, a fin de resolver litigios con efectos legales. Este criterio es más amplio y claro que el de la comunidad andina.

También en la doctrina se discute si los árbitros pueden tener legitimación activa. El profesor Manuel Pachón Muñoz (1998) sostiene que los árbitros deben tener legitimación activa, criterio además corroborado por el profesor Hernando Morales Molina. A mi criterio, para poder definir esta interrogante habría que analizar las normas internas de cada país y determinar si la jurisdicción

\footnotetext{
${ }^{9}$ Proceso I-93, del TJCA 
arbitral consta dentro de la jurisdiccional, sólo así sería aceptable tal criterio.

Sobre lo que no existe duda, es que en el recurso de interpretación prejudicial no hay legitimidad pasiva, toda vez que es un procedimiento de cooperación entre el Juez Comunitario y los jueces nacionales. De ahí, que la doctrina establece que no es un recurso, sino un mecanismo de cooperación.

\section{El Reenvío y Cooperación entre los Jueces.}

Lo primero que hay que aclarar es que el artículo 33 del TCTJCA no establece en qué procesos procede la solicitud de interpretación prejudicial. Esto puede ocasionar un grave problema, y simplemente tendríamos que concluir que es en cualquier proceso donde se presente controversia en la aplicación de una norma andina. También la doctrina es unánime en sostener que si ya ha habido interpretación prejudicial sobre una norma comunitaria específica, no cabe solicitar nuevamente interpretación sobre la misma. Sin embargo el profesor Perotti sostiene que esta teoría ha sido rechazada en forma expresa por el TJCA en el precedente Aktiebolaget Volvo, en el cual sostuvo que 'la interpretación que en su sentencia establezca el Tribunal comunitario, rige tan sólo para el caso objeto de la consulta $y$, por tanto, no exime al juez nacional de la obligación de consultar en casos similares o análogos». Por tanto la obligatoriedad de la remisión prejudicial existe con independencia de que el Tribunal Andino se haya expedido anteriormente sobre la hermenéutica que debe a- tribuirse a la disposición en cuestión.

Donde si hay coincidencia entre la doctrina y el TJCA es que en el evento que haya controversia en la aplicación de una norma andina, así el juez piense que la norma es clara en su sentido, tiene la obligación de solicitar la interpretación prejudicial. En consecuencia, la remisión a la jurisdicción supranacional es imperativa en todos los casos en los cuales se presente una "cuestión de interpretación" del derecho comunitario, y ello a pesar de la "claridad" de la disposición.

El TJCA, sobre el tema ha establecido lo siguiente:

Es evidente que el juez nacional es quien debe determinar si se requiere o no la interpretación prejudicial, pero tal determinación no es arbitraria y debe hacerse con pleno conocimiento de causa, ya que, según se desprende del citado artículo 29 del Tratado del Tribunal, sería improcedente la interpretación de normas comunitarias cuya aplicación no resulte necesaria, según los términos en los que se haya planteado la litis.

Finalmente, nótese que el artículo 123 del Estatuto del TJCA, establece la facul tad de las partes de solicitar al Juez nacional de la Causa que realice la solicitud de interpretación prejudicial, pero obviamente siempre y cuando sea la instancia final.

El artículo 125 del Estatuto del TJCA, establece las condiciones y requisitos que debe contener la solicitud de interpreta- 
tación prejudicial. Al respecto, pasaremos a enunciar y comentar tales condiciones:

a) El nombre e instancia del juez o tribunal nacional consultante;

b) La relación de las normas del ordenamiento jurídico de la Comunidad Andina cuya interpretación se requiere;

c) La identificación de la causa que origine la solicitud;

d) El informe sucinto de los hechos que el solicitante considere relevantes para la interpretación; y,

e) El lugar y dirección en que el juez o tribunal recibirá la respuesta a su consulta.

Sobre el requisito descrito en el literal "d" anterior, la profesora Samanta Sánchez plantea la siguiente preocupación:

En el caso de la interpretación prejudicial, nos encontramos con una limitación, al menos teórica, que excluye del análisis del juez, a los hechos del caso que genera la solicitud de interpretación. Y decimos teórica, porque si bien en las disposiciones del Tratado que crea el TJCA se recalca que la competencia del juez comunitario es exclusivamente sobre el derecho comunitario (excluyendo expresamente a los hechos); el mismo Tratado contempla, entre los requisitos de la solicitud, un resumen de los hechos, a efectos de ubicar al juez comunitario en el caso, e impedir que la interpretación prejudicial sea tan abstracta que no sirva su propósito de instruir al juez nacional en la resolución del caso.
Este conocimiento de los hechos, aunque sea somero, y a pesar de que el juez comunitario no pueda cuestionarlos, sino aceptarlos tal y como se los presenta el juez nacional, implica, a nuestro modo de ver, que en realidad la interpretación prejudicial no es tan alejada de los hechos ni por lo tanto tan abstracta como se argumenta y por ello se asemeja en gran medida a la interpretación judicial, donde los hechos dan una autenticidad y una concretización a la labor del juez, que es lo que le otorga su validez. Y es que en definitiva, el juez comunitario hace una interpretación jurídica de los hechos relevantes que se le presentan, no los califica en principio, pero de ellos extrae los elementos pertinentes del caso, para luego subsumir esos hechos en las normas comunitarias que va a interpretar para el caso concreto.

Y la preocupación la considero cierta, resulta muy importante la separación de los "hechos" del "derecho". Sobre los hechos, la prueba de fuego será la imparcialidad del juez solicitante toda vez que si bien el juez comunitario no puede calificarlos, es indudable que la interpretación no puede abstraerse de los mismos.

Este criterio se confirma de la lectura del artículo 126 del Estatuto del TJCA que establece:

Recibida la solicitud de consulta, el Secretario la sellará, dejará constancia en ella de la fecha de presentación o recepción, y la remitirá al Presidente 
para su consideración por el Tribunal dentro del término de treinta días siguientes al de la admisión de la solicitud por el Tribunal, éste dictará sentencia. En su interpretación, el Tribunal deberá limitarse a precisar el contenido y alcance de las normas que conforman el ordenamiento jurídico de la Comunidad Andina, referidas al caso concreto. El Tribunal no interpretará el contenido y alcance del derecho nacional ni calificará los hechos materia del proceso, pero podrá referirse a éstos cuando ello sea indispensable a los efectos de la interpretación solicitada.

Finalmente, sobre la oportunidad de la solicitud de interpretación prejudicial el TJCA ha establecido:

En este caso y en los demás -sea la consulta opcional o facultativa, o si siendo obligatoria, el proceso aún no se encuentra en la etapa de decisión- la consulta prejudicial puede solicitarse en cualquier tiempo. Resulta recomendable entonces que se formule cuanto antes a fin de evitar dilaciones inútiles. ${ }^{10}$

\section{Reconocimiento de la Interpretación Prejudicial en el Derecho Nacional.}

Si bien la Constitución Ecuatoriana de manera expresa propugna la integración, de manera especial la andina y latinoamericana ${ }^{11}$, y que además reconoce y respeta plenamente al Derecho Internacional, considero que la próxima aprobación de una nueva Constitución en el país es una oportunidad para realizar ciertas puntualizaciones en torno al Derecho Andino, y en particular sobre la interpretación prejudicial.

La interpretación prejudicial como sistema de cooperación judicial tendiente a obtener la uniformidad de la aplicación de las normas comunitarias, a mi criterio, requiere un reconocimiento expreso constitucional toda vez que -de una y otra manera- en el ejercicio de las competencias de la administración de justicia participan jueces ajenos a la función judicial (jueces comunitarios) cuyo criterio de interpretación es de obligatorio acatamiento para el Juez Nacional en el caso específico que juzga. En este sentido, el reconocimiento constitucional de esta figura, fortalecería plenamente la certeza y seguridad jurídica de los jueces nacionales y sin duda, afianzaría aún más la aplicación uniforme de las normas comunitarias.

Por tanto, mi recomendación en este sentido, es que en el capítulo constitucional de la Función Judicial se reconozca expresamente este sistema de cooperación judicial.

Análisis de la interpretación Prejudicial, dentro del Proceso 154-Ip-2006, de los Artículos 134, 136 Literal a) y 150 de la Decisión 486 de la Comisión de la Comunidad Andina Solicitada por el Concejo de Estado de la República de Colombia.

${ }^{10}$ Proceso No. 1-IP-87, del TJCA

${ }^{11}$ Artículo 4 CPE, numeral 5.

12

PODIUM No. 31, Guayaquil, Junio 2017, pp. 1-19

Universidad Espíritu Santo - UEES

ISSN: 1390 - 5473 


\section{Actor: Sociedad Burguer King Corpo- ration \\ Marca: BURGUER KING}

\section{Introducción sobre las Marcas.}

Antes de entrar al análisis del caso referido, es necesario indicar que la decisión 486 de la comisión de la Comunidad Andina se aprobó en Lima el 14 de Septiembre de 2000 y según lo dispuesto en la misma decisión en su Art. 274, entró en vigencia a partir del $1^{\circ}$ de diciembre de 2000 y sustituyó la decisión 344, norma que estuvo vigente desde el $1^{\circ}$ de enero de 1.994.

Dicha decisión (486) que regula la Propiedad Industrial dentro de los países de la Comunidad Andina según sostiene la doctrina fue el resultado de varias reuniones de expertos, delegados de cada uno de los países miembros, que con la ayuda de la OMPI (Organización Mundial de la Propiedad Intelectual), elaboraron la propuesta. Una gran parte de las solicitudes de interpretación prejudicial, han sido sobre las normas de la citada Decisión 486 (y sobre la anterior Decisión 344).

El concepto de marca ha sid o ampliamente desarrollado por la doctrina y por la jurisprudencia. Dentro de aquella, la definición aportada por el tratadista Hermenegildo BAYLOS CORROZA (1978) se encuentra concebida en los siguientes términos: "La marca es un signo destinado a individualizar los pro- ductos o los servicios de una empresa determinada y hacer que sean reconocidos en el mercado por el público consumidor" (p. 581).

El TJCA ${ }^{12}$ ha sostenido con relación a las marcas las siguientes caracterísicas:

Tal como aparecen enumerados en el primer párrafo del artículo 81 de la citada Decisión 344 (hoy fuera de vigencia), estos han sido desarrollados en numerosas sentencias, entre otras las referentes a los casos de interpretación prejudicial Nos. 27-IP-95 (G.O. No 257 de 14 de abril de 1997), 22-IP-96 (G.O. No 265 de 16 de mayo de 1997) y 15-IP-97 (G.O. $\mathrm{N}^{\mathrm{o}} 314$ de 18 de diciembre de 1997). Ese desarrollo jurisprudencial revela que: a) La Perceptibilidad guarda relación con todo elemento, signo o indicación apreciable por los sentidos y que pueda ser aprehendido por el público. b) La Distintividad, característica esencial de la marca, tiene como función diferenciar productos o servicios producidos o comercializados por una persona, de los idénticos o similares provenientes de otra, logrando de esta manera que el consumidor, destinatario final del producto al que la marca se refiere, pueda llegar a diferenciarlos. c) La Representación Gráfica. Esta característica del signo permite transmitir en forma material el contenido y las dimensiones del mismo y, posteriormente, la publicación que la norma exige, destinada

${ }^{12}$ Interpretación 60-IP-2001, TJCA 
esta a que los competidores puedan conocer y apreciar dicho signo para establecer las diferencias o semejanzas con el ya registrado o solicitado para su registro, y proceder si fuere el caso a la impugnación del mismo a través del incidente de las observaciones previsto en la norma andina. Resta precisar que un signo que no reuniere esas tres características, mal podría ser protegido por un registro marcario.

\section{Exposición del Caso Analizado (Proceso 154-IP-2006).}

Burguer King Corporation, plantea que se declare la nulidad de la Resolución No-033098, del 30 de diciembre del 2004, emitida por el Jefe de la División de Signos Distintivos de la Superintendencia de Industrias y Comercio de Colombia, mediante la cual se resolvió: Declarar infundada la oposición formulada por la sociedad BURGER KING CORPORATION y conceder el registro de la marca mixta SUPPER KING (mixta), para distinguir: "Servicio de Restaurante (alimentación)"; servicios comprendidos en la clase 43 de la Clasificación Internacional de Niza, octava edición; a favor del señor José Rodrigo Lievano M., por el término de diez (10) años contados a partir de la fecha de la presente resolución. De igual forma se impugnaron las Resoluciones de los Órganos Superiores de alzada que ratifi- caron la citada Resolución.

La demanda se fundamenta en:

a) que ambas marcas son similarmente confundibles; b) que la Marca BURGUER KING es notoriamente conocida; c) que los signos son casi idénticos, causando error y confusión en el consumidor; d) que en ambas marcas la expresión predominante es la palabra "KING": e) que BURGUER KING fue previamente registrada; f) que ambas marcas guardan el mismo vínculo con los productos y servicios; g) que ambas marcas tienen idénticos canales de publicidad y comercialización y h) que no hubo un adecuado examen de registrabilidad.

La contestación a la demanda del Consejo de Estado Colombiano se fundamenta en: a) la marca SUPPER KING tiene la suficiente fuerza distintiva; b) que las marcas tienen elementos diferenciadores por lo que pueden coexistir en el mercado; c) que no produce a error o Confusión en el consumidor; d) que los actos administrativos impugnados no son nulos ya que se ajustan a las normas del derecho nacional colombiano.

Las normas comunitarias que se solicitan sean interpretadas son la interpretación prejudicial de los artículos 134, 136 literal a) y 150 de la Decisión 486 de la Comisión de la Comunidad Andina. ${ }^{13}$

\footnotetext{
13 "Artículo 134.- A efectos de este régimen constituirá marca cualquier signo que sea apto para distinguir productos o servicios en el mercado. Podrán registrarse como marcas los signos susceptibles de representación gráfica. La naturaleza del producto o servicio al cual se ha de aplicar una marca en ningún caso será obstáculo para su registro. Podrán constituir marcas, entre otros, los siguientes signos:
} 


\section{Principales Conclusiones del Tribu- nal de Justicia de la Comunidad Andina en el caso analizado.}

De la Resolución del TJCA en el presente caso, en su forma quiero destacar lo siguiente:

- $\quad$ El Tribunal confirma el cumplimiento de las condiciones previstas en el artículo 125 del Estatuto del Tribunal que se refieren a los requisitos de la solicitud de interpretación.

- El Juez nacional separó los "hechos relevantes" del derecho, con imparcialidad, de tal forma que el Tribunal tuvo los elementos necesarios para la interpretación.
- El Tribunal confirma que las normas que se solicitan sean interpretadas forman parte del ordenamiento jurídico de la Comunidad Andina.

- El Tribunal presenta las bases jurídicas que lo facultan a realizar la interpretación prejudicial.

- $\quad$ No se deja aclarado si la consulta es facultativa u obligatoria. Del estudio del fallo, da la impresión que el caso se encuentra en última instancia por lo que sería de las llamadas "consultas obligatorias".

- $\quad$ El Tribunal sustenta la interpretación en el análisis doctrinal y de la normativa comunitaria sobre los siguientes temas: la marca y sus elementos constitutivos; clases de marcas; impedimentos

\footnotetext{
${ }^{13}$ Continuación página anterior

a) las palabras o combinación de palabras;

b) las imágenes, figuras, símbolos, gráficos, logotipos, monogramas, retratos, etiquetas, emblemas y escudos;
c) los sonidos y los olores;
d) las letras y los números;
e) un color delimitado por una forma, o una combinación de colores;
f) la forma de los productos, sus envases o envolturas;
g) cualquier combinación de los signos o medios indicados en los apartados anteriores”.

“Artículo 136.- No podrán registrarse como marcas aquellos signos cuyo uso en el comercio afectara indebidamente un derecho de tercero, en particular cuando:

a) sean idénticos o se asemejen, a una marca anteriormente solicitada para registro o registrada por un tercero, para los mismos productos o servicios, o para productos o servicios respecto de los cuales el uso de la marca pueda causar un riesgo de confusión o de asociación;

h) constituyan una reproducción, imitación, traducción, transliteración o transcripción, total o parcial, de un signo distintivo notoriamente conocido cuyo titular sea un tercero, cualesquiera que sean los productos o servicios a los que se aplique el signo, cuando su uso fuese susceptible de causar un riesgo de confusión o de asociación con ese tercero o con sus productos o servicios; un aprovechamiento injusto del prestigio del signo; o la dilución de su fuerza distintiva o de su valor comercial o publicitario".

“Artículo 150.- Vencido el plazo establecido en el artículo 148, o si no se hubiesen presentado oposiciones, la oficina nacional competente procederá a realizar el examen de registrabilidad. En caso se hubiesen presentado oposiciones, la oficina nacional competente se pronunciará sobre éstas y sobre la concesión o denegatoria del registro de la marca mediante resolución". 
para el registro de una marca; riesgo de confusión; reglas para realizar el cotejo marcario; signos en idioma extranjero y palabras de uso común; la marca notoria y su prueba; la imitación o reproducción de una signo notoriamente conocido; la conexión competitiva y sobre el procedimiento para el examen de registrabilidad. - De lo anterior se colige, que el Juez Comunitario entrega al Juez Nacional en abundancia los criterios doctrinales, normativos y jurisprudenciales, que le permitan tomar la adecuada resolución en el caso concreto, concretándose la cooperación en la actuación jurisdiccional.

Sobre el tema de fondo, destaco de la resolución analizada los siguientes preceptos que se desprenden de las conclusiones:

- Una marca puede ser registrada cuando se distingue de productos o servicios en el mercado y si cumple el requisito de representación gráfica.

- Los signos denominativos compuestos son los integrados por dos palabras, con o sin significación conceptual

- $\quad$ En la marca mixta, es fundamental establecer cuál es el elemento que prevalece sobre el otro en la mente del consumidor, a través del examen de registrabilidad.

- $\quad$ No son registrables los signos que de acuerdo a las normas comunitarias sean idénticos o semejantes a una marca anteriormente registrada para los mismos servicios o productos, evitando causar confusión o error al consumidor.
- $\quad$ Sólo el riesgo de error y confusión en el consumidor ya configura la prohibición de registro.

- $\quad$ Si un signo es integrado por palabras en idioma extranjero, o si su significado ha hecho del conocimiento de la mayoría del público consumidor o usuario, la denominación no será registrable.

- La marca notoriamente conocida es la que adquiere esa calidad porque se encuentra ampliamente difundida entre una colectividad de individuos y ha logrado el reconocimiento del mismo. La notoriedad no se presume, debe probarse, y esa calidad no le otorga por el hecho de estar registrada en varios países.

- $\quad$ No se puede registrar como marca los signos que constituyan la reproducción, imitación, traducción, transcripción total o parcial de un signo distintivo notoriamente conocido en el país o en el comercio subregional, o internacional.

- Para llegar a determinar la similitud entre las dos marcas, se ha de considerar los criterios de conexión competitiva existente entre los productos y servicios amparados por las mismas.

- $\quad$ Obligatoriamente se debe realizar el examen de registrabilidad en forma previa a otorgar un registro, debiendo notificar al solicitante la decisión debidamente fundamentada.

Concluye la interpretación estableciendo que le corresponde al Juez nacional colombiano dictar el fallo correspondiente para lo cual deberá considerar obligatoriamente la interpretación realizada.

Este fallo es ejemplarizador de la 
materialización de la cooperación de los Jueces Comunitarios y los Jueces Andinos, en un caso concreto.

\section{Conclusiones Finales}

Las conclusiones a las que he llegado como producto del presente trabajo de investigación y estudio, son las siguientes:

- En los procesos de integración como el Andino, es fundamental contar con un órgano jurisdiccional que dirima la validez de los actos amparados en normas comunitarias así como emita las interpretaciones que sobre dichas normas los casos concretos exijan.

- El recurso de Interpretación Prejudicial tiene como finalidad principal garantizar la aplicación uniforme de las normas comunitarias en todos los países miembros de la Comunidad Andina, por tanto es acertada la obligatoriedad de aplicación por parte de los jueces nacionales de las conclusiones interpretatorias. - $\quad$ En la comunidad europea, además de la interpretación las normas comunitarias, la interpretación prejudicial tiene también como finalidad el "control de validez" de los actos de las instituciones europeas, por eso es considerada como otro medio de protección de los derechos ciudadanos.

- $\quad$ Para este fin, el método adecuado es la cooperación de los jueces comunitarios con los jueces nacionales, convirtiéndose en actores paralelos en el ejercicio de las competencias jurisdiccionales.
- La interpretación no es propiamente un recurso, sino un procedimiento de cooperación entre el Juez comunitario y los tribunales nacionales

- Dentro de la Estructura de la Comunidad Andina, resulta lógico que la interpretación prejudicial sea competencia del Tribunal de Justicia.

- El Tribunal de Justicia de la CA tiene competencia exclusiva para la interpretación de las normas comunitarias, pero a su vez tiene prohibición expresa de interpretación de normas locales y de los hechos y actos relevantes del proceso.

- El TJCA no tiene competencias para resolver sobre el proceso principal ya que está es competencia de los jueces nacionales.

- Las normas que conforman el sistema jurídico de la Comunidad Andina están plenamente determinadas en el Tratado de Creación del TJCA.

- Es necesario que el legislador andino, a través del instrumento adecuado, aclare ciertos conceptos relacionados con el recurso de interpretación prejudicial, a saber: ¿qué se entiende por "recurso" para efectos de determinar la obligatoriedad o no de la interpretación?; i sobre qué tipos de procesos cabe solicitar la interpretación, sobre todos los procesos?; ¿los órganos jurisdiccionales de cada país se determinan por el derecho nacional o el TJCA tiene autonomía para decidir si un órgano forma parte o no? Estos entre los principales temas por clarificar.

- Varios de las interpretaciones estudiadas, a saber casos sobre las Mar- 
cas ROLEX, VOLVO, BURGUERKING, MC DONALDS coincidían en las normas sobre las cuales se solicitó la interpretación. Pregunto entonces: ¿Será necesario establecer la facultad de que el Tribunal pueda emitir interpretaciones de carácter general y obligatorio, de tal forma de no congestionar innecesariamente a los magistrados?. A mi criterio sí. - $\quad$ Es evidente la superioridad jerárquica de las normas comunitarias sobre las nacionales, siendo los magistrados del TJCA los garantes de este principio de supranacionalidad de las normas comunitarias.

- $\quad$ Si bien la interpretación prejudicial debe separar los "hechos" de los "derechos", en la práctica es teórico, ya que de los hechos se extrae también elementos fundamentales para realizar una adecuada interpretación de las normas comunitarias.

- Para efectos de la interpretación, tiene que aplicarse todos los métodos ofrecidos por la Filosofía y la hermenéutica jurídica, como el gramatical, histórico, sistemático, lógico jurídico, etc, ya que la interpretación jurídica completa es el resultado de un ejercicio global de esos métodos.

- $\quad$ El estudio del derecho comunitario resulta apasionante, y constantemente estará sujeto a grandes desafíos de engranaje entre las normas comunitarias y las normas nacionales, órganos comunitarios y órganos locales, siempre pensando que este es el camino al desarrollo económico y social de los países participantes de un proceso de integración, cuyo objetivo máximo debe inspirarse en el interés general y la mejora de la calidad de vida de los ciudadanos.

\section{Referencias}

Borchardt, K.-D. (2011). El ABC del Derecho de la Unión Europea. Luxemburgo: Oficina de Publicaciones Comisión Europea.

Comunidad Andina. (2010). Recuperado el 31 de 03 de 2017, de http://www.comunidadandina.org/

Consejo Andino. (22 de 06 de 2001). Tribunal Andino. Recuperado el 31 de 03 de 2017, de Estatuto del Tribunal de Justicia de la Comunidad Andina: http://www.tribunalandino.org.ec/sitetjca1/T_Estatut $\quad 0 \quad \% \quad 2 \quad 0 \quad$ del $\% 20$ Tribunal $\% 20 \mathrm{de} \% 20$ Justicia $\% 20 \mathrm{de} \% 201 \mathrm{la} \% 20$ Comunidad $\% 20 \mathrm{~A}$ ndina.pdf

Corroza, B. (1978). Tratado de Derecho Industrial (Primera ed.). Madrid: Civitas.

Diez-Moreno, F. (2005). Manual de Dererecho de la Unión Europea (Quinta ed.). Thomson Reuters.

Guy, I. (1995). Manual del Derecho Comunitario General (Tercera ed.). Barcelona: Ariel.

Mangas, A., \& Liñán, D. (2017). Institucio- 
nes y Derecho de la Unión Europea. Tecnos.

Perotti, A. (05 de Marzo de 2001). Comunidad Andina. (B. D. Andina, Ed.) Recuperado el 07 de 03 de 2017, de Algunas Consideraciones sobre la Interpretación Prejudicial Obligatoria en el Derecho Andino: http://www.comunidadandina.org/bda/docs/CAN-INT-0010.pdf

Renifo, E. (1996). Propiedad Intelectual: El Moderno Derecho de Autor. Bogotá: Universidad Externado de Colombia.

Sánchez, S. (2010). Interpretación del derecho comunitario: La interpretación prejudicial. Publicaciones Jurídicas Venezonalas, 22.

Tribunal Andino. (03 de 10 de 1996). Tratado de Creación del Tribunal de Justicia de la Comunidad Andina, con el protocolo modificatorio de Cochabamba. Recuperado el 01 de 04 de 2017, de http://www.tribunalandino.org.ec/sitetjca1/TCREACION.pdf

Tribunal Andino. (1998). Interpretación Prejudicial del artìculo 83 Literal a) de la Decisión 344.
Tribunal de Justicia de la Comunidad Andina. (26 de 05 de 1969). Acuerdo de Cartagena. Cartagena de Indias, Colombia.

Tribunal de Justicia de la Comunidad Andina. (2006). Interpretación Prejudicial del Tribunal Andino de Justicia. Caso Burger King. 